HIAS-E-78

\title{
Skill, Value and Remuneration in Committees
}

\author{
Ruth Ben-Yashar ${ }^{(a)}$ and Shmuel Nitzan ${ }^{(a),(b)}$
}

(a) Department of Economics, Bar Ilan University, Ramat Gan 52900, Israel

(b) Hitotsubashi Institute for Advanced Study, Hitotsubashi University, Tokyo, Japan

November, 2018

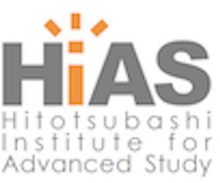

Hitotsubashi Institute for Advanced Study, Hitotsubashi University

2-1, Naka, Kunitachi, Tokyo 186-8601, Japan

tel:+81 425808604 http://hias.ad.hit-u.ac.jp/

HIAS discussion papers can be downloaded without charge from: http://hdl.handle.net/10086/27202

https://ideas.repec.org/s/hit/hiasdp.html

All rights reserved. 


\title{
Skill, Value and Remuneration in Committees
}

by

\author{
Ruth Ben-Yashar ${ }^{a}$ and Shmuel Nitzan ${ }^{b}$.
}

\begin{abstract}
Relatively modest difference in individual decisional skills may warrant substantial inequality in personal remunerations. This implies that the return on investment in decisional skills may be very high. These claims are illustrated applying the uncertain dichotomous choice setting while clarifying the complex relationship between the individual skills, the individual optimal decisional weights and the skill-dependent values, i.e., the individual probabilities to be pivotal.
\end{abstract}

Keywords: decisional skills, optimal decisional weights, skill-dependent power, remuneration.

Classification Codes: D7.

a Department of Economics, Bar Ilan University, Ramat Gan 52900, Israel.

${ }^{\mathrm{b}}$ Department of Economics, Bar Ilan University, Ramat Gan 52900, Israel and

Hitotsubashi Institute for Advanced Study, Hitotsubashi University, Tokyo, Japan . 


\section{Introduction}

In standard neoclassical economics, heterogeneous inputs deserve differential payments. This basic principle applies to decision makers who are members of a group that makes collective decisions under uncertainty. In this context, however, an individual's contribution to the collective decision depends on his and the other group members' decisional skills that determine the optimal group decision rule. Given this optimal rule, the group members' probabilities of being pivotal and unequivocally contribute to the collective decision can be calculated and, in turn, the warranted remunerations.

This letter presents a new notion of an individual's skill-dependent value (power), i.e., the probability that he is pivotal, taking into account not only the relative number of the decision profiles enabling the decision maker to be pivotal, but also the different probabilities of these profiles. Applying the basic uncertain dichotomous choice setting, the skill-dependent value is calculated under the optimal group decision rule. It is shown that even modest heterogeneity in individual decisional skills may warrant substantial inequality in personal remuneration of the group members. In other words, one should not underestimate the effect of relatively small variations in the decisional skills of the group members. Such variations may result in an optimal weighted majority rule that gives rise to very unequal warranted remunerations. This finding sheds new light on the old and still ongoing debate regarding the possibility of justifying extreme unequal payments within groups of professionals of seemingly inconsequential diverse personal qualifications. It implies that, the marginal benefit of investment in skill can be very high.

\section{The model}

Consider an n-member committee that confronts two alternatives, 1 and -1 , one of which is correct and therefore better for all decision makers. The two alternatives are symmetric i.e., the priors of the two states of nature determining whether an alternative is correct or incorrect are equal and the net benefits of a correct decision under the two states of nature are equal. The identity of the better alternative is unknown. Every decision maker selects one of the two alternatives. We denote by $x_{i}$ 
individual i's decision, where $x_{i}=1$ or $x_{i}=-1$. Decision maker $i$ chooses the correct alternative with probability $p_{i}$, which reflects his decisional skill (competence). We assume independent decisional competencies and that $1 / 2<p_{i}<1$. With no loss of generality, it is assumed that $\mathrm{i}<\mathrm{j}$ implies that $p_{i} \geq p_{j}$, that is, committee members are ordered by their skills. Hence, $x_{l}$ is the decision of the most competent committee member. The collective decision is based on the decisions of the individuals and is made by a decisive aggregation rule $f$ that assigns 1 or -1 to any decision profile $\left(x_{1}\right.$, $\left.x_{2, \ldots,} x_{n}\right) .^{1}$

\section{The optimal decision rule}

The collective decision rule that optimizes the decision making process, i.e., maximizes the collective probability of making a correct decision, is a weighted majority rule (WMR), Nitzan and Paroush (1982) and Shapley and Grofman (1984). Specifically, the optimal aggregation rule is $\operatorname{sign}\left(w^{*}{ }_{1} x_{1+} \ldots+w_{n}{ }_{n} x_{n}\right)$, where $w^{*}{ }_{i}=$ $\ln \left[p_{i} /\left(1-p_{i}\right)\right]$ and $\operatorname{sign}(m)=1$, if $m>0$ and -1 otherwise.

In committees, the number of the potentially optimal (efficient and, therefore, economically relevant) weighted majority rules is surprisingly small. In particular, in committees of three, four and five-members there exist, respectively, only two, three and seven such rules. The systems of weights $w_{i}=\left(w_{1}, w_{2}, w_{3}, w_{4}, w_{5}\right)$, $\mathrm{i}=\{a, b, c, d, e, f, g\}$, such that the weights are ordered by skill (that is, $w_{1} \geq w_{2} \geq$ $w_{3} \geq w_{4} \geq w_{5}$ ), defining these seven different weighted majority rules for a fivemember group are:

$f_{a}$ (the expert rule) defined by $w_{a}=(1,0,0,0,0)$.

$f_{b}$ defined by $w_{b}=(1,1,1,0,0)$.

$f_{c}$ defined by $w_{c}=(2,1,1,1,0)$.

$f_{d}$ (simple majority rule) defined by $w_{d}=(1,1,1,1,1)$.

$f_{e}$ defined by $w_{e}=(3,1,1,1,1)$.

$f_{f}$ defined by $w_{f}=(2,2,1,1,1)$.

$f_{g}$ defined by $w_{g}=(3,2,2,1,1)$.

${ }^{1}$ See Austen-Smith and Banks (1996), Ben-Yashar and Nitzan (1997), Dietrich and List (2013), Young (1988) and recently, Nitzan and Paroush (2018). 
Notice that, for a five-member group, any possible system of optimal weights is equivalent to one of the above seven systems of weights.

\section{The standard power of committee members}

A plausible measure of the value of a decision maker is his probability of determining the collective decision, i.e., the probability of being pivotal. If all decision profiles are equally likely, as usually assumed in the literature, an individual's power is the fraction of all his swing decisions ${ }^{2}$. For a five-member committee decision rules, the possible standard power distributions are listed in Table 1 . In a similar way, it is possible to derive the power distributions for 3 and 4-member committees. For example, in the case of the WMR defined by $(3,1,1,1,1)$, the most competent group member, individual 1, is pivotal in 28 decision profiles and each of the remaining members is pivotal in 4 profiles. After normalization, we therefore get that the standard power distribution in this case is $(63.64,9.09,9.09,9.09,9.09)$.

Table 1: Weights and standard power

\begin{tabular}{|l|l|}
\hline weights & $\begin{array}{l}\text { standard power } \\
\text { (normalized) }\end{array}$ \\
\hline$w_{a}=(1,0,0,0,0)$ & $(100,0,0,0,0)$ \\
\hline$w_{b}=(1,1,1,0,0)$ & $(33.33,33.33,33.33,0,0)$ \\
\hline$w_{c}=(2,1,1,1,0)$ & $(50,16.67,16.67,16.67,0)$ \\
\hline$w_{d}=(1,1,1,1,1)$ & $(20,20,20,20,20)$ \\
\hline$w_{e}=(3,1,1,1,1)$ & $(63.64,9.09,9.09,9.09,9.09)$ \\
\hline$w_{f}=(2,2,1,1,1)$ & $(28.57,28.57,14.29,14.29,14.29)$ \\
\hline$w_{g}=(3,2,2,1,1)$ & $(38.46,23.08,23.08,7.69,7.69)$ \\
\hline
\end{tabular}

${ }^{2}$ In the context of simple games, the same idea motivates the power index proposed by Banzhaf (1965). 
Table 1 clarifies that different decisional weights result in standard power inequality.

\section{The skill-dependent power of committee members}

The identity of the optimal rule and, in turn, of the group members' power/value hinges on their skills. First, the skills determine the identity of the optimal weighted majority rule. Second, given the group members' optimal weights, the skills determine the probability of an individual to be pivotal, i.e., the probability of the decision profiles of the other $(n-1)$ members that enable him to determine the collective decision.

To illustrate and clarify the meaning of skill-dependent power, consider the simplest setting of a three-member group, $n=3$, where the skills of the group members are given by $(0.850 .80 .6)$. In this case, the optimal weighted majority rule is simple majority. ${ }^{3}$ Under this rule, every decision maker is pivotal in the two situations where the votes of the other two group members are divided. However, now taking into account the information about decisional skills, the probability of each of the three differently skilled committee members to be pivotal is no longer the same. It depends on the decisional skills of the other two members as follows:

Individual 1' power: $0.8 * 0.4+0.2 * 0.6=0.44$

Individual 2 ' power: $0.85 * 0.4+0.15 * 0.6=0.43$

Individual 3 ' power: $0.85 * 0.2+0.15 * 0.8=0.29$

So the normalized distribution of skill-dependent power becomes $(0.379,0.3706$, $0.25)$.

Table 2 illustrates seven possible skill profiles that result in all the possible optimal weighted majority rules and their corresponding distributions of skilldependent power/value.

\footnotetext{
${ }^{3}$ The only other potentially optimal but inferior rule is the expert rule.
} 
Table 2: Skills, weights and power

\begin{tabular}{|c|c|c|c|}
\hline $\begin{array}{l}\text { Decisional skills } \\
\left(p_{1}, \ldots, p_{5}\right)\end{array}$ & $\begin{array}{l}\text { Optimal weights } \\
\left(w^{*}{ }_{1}, \ldots, w_{5}^{*}\right)\end{array}$ & $\begin{array}{l}\text { optimal weights } \\
\text { (Normalized) }\end{array}$ & $\begin{array}{l}\text { standard power } \\
\text { skill-dependent power } \\
\text { (Normalized) }\end{array}$ \\
\hline$(0.8,0.7,0.6,0.5,0.5)$ & $(1.386,0.847,0.405,0,0)$ & $w_{a}=(1,0,0,0,0)$ & $\begin{array}{l}(100,0,0,0,0) \\
(100,0,0,0,0)\end{array}$ \\
\hline$(0.8,0.75,0.6,0.5,0.5)$ & $(1.386,1.098,0.405,0,0)$ & $w_{b}=(1,1,1,0,0)$ & $\begin{array}{l}(33.33,33.33,33.33,0,0) \\
(36.29,35.48,28.22,0,0)\end{array}$ \\
\hline$(0.8,0.75,0.6,0.54,0.5)$ & $(1.386,1.098,0.405,0.157,0)$ & $w_{c}=(2,1,1,1,0)$ & $\begin{array}{l}(50,16.67,16.67,16.67,0) \\
(56.16,16.75,13.66,13.42,0)\end{array}$ \\
\hline$(0.8,0.7,0.7,0.7,0.64)$ & $(1.386,0.847,0.847,0.847,0.574)$ & $w_{d}=(1,1,1,1,1)$ & $\begin{array}{l}(20,20,20,20,20) \\
(21.73,19.89,19.89,19.89,18.6)\end{array}$ \\
\hline$(0.8,0,7,0.6,0.54,0.54)$ & $(1.386,0.847,0.405,0.157,0.157)$ & $w_{e}=(3,1,1,1,1)$ & $\begin{array}{l}(63.64,9.09,9.09,9.09,9.09) \\
(69.57,8.38,7.41,7.31,7.31)\end{array}$ \\
\hline$(0.8,0.75,0.6,0.6,0.58)$ & $(1.386,1.098,0.405,0.405,0.322)$ & $w_{f}=(2,2,1,1,1)$ & $\begin{array}{l}(28.57,28.57,14.29,14.29,14.29) \\
(31.8,30.78,12.5,12.5,12.4)\end{array}$ \\
\hline$(0.8,0.75,0.6,0.54,0.54)$ & $(1.386,1.098,0.405,0.157,0.157)$ & $w_{g}=(3,2,2,1,1)$ & $\begin{array}{l}(38.46,23.08,23.08,7.69,7.69) \\
(43.36,24.24,19.61,6.38,6.38)\end{array}$ \\
\hline
\end{tabular}

\subsection{Modest skill heterogeneity may warrant very unequal remunerations}

Table 2 provides an unequivocal positive answer to the question "can unequal pay be justified given modest variability in the decisional skills of the committee members". For example, in row five, the skills $(0.8,0,7,0.6,0.54,0.54)$ give rise to the optimal weights $\left(w^{*} 1, \ldots, w^{*}\right)=(1.386,0.847,0.405,0.157,0.157)$ that are equivalent to the optimal normalized system of weights $w_{e}=(3,1,1,1,1)$. These weights justify the normalized standard power distribution $(63.64,9.09,9.09,9.09,9.09)$ or the normalized 
skill-dependent power distribution (69.57,8.38,7.41,7.31,7.31). In this case, the difference between the skills of individuals 1 and 2 is just 0.1 , however 1 deserves a pay which is seven times larger than that of 2 and of the other members according to the standard power and a pay which is more than 8 (9) times larger than that of individual 2 (3, 4 or 5$)$ according to the skill-dependent power. The effect of using skill-dependent power instead of standard power intensifies the power inequality and, in turn, the warranted payment inequality. In row four, the optimal decision rule corresponding to the skills $(0.8,0.7,0.7,0.7,0.64)$ is the simple majority defined by equal decisional weights. In turn, the decision makers are assigned equal optimal decisional weights and therefore the standard power distribution is uniform. Nevertheless, the skill-dependent power distribution is $(21.73,19.89,19.89,19.89,18.6)$ reflecting the difference in the individual skills. To sum up, the application of skilldependent power justifies increased payment inequality.

\subsection{More on the relationship between skills and payment}

Table 2 suggests that investment in individual decisional competence can yield substantial marginal benefit. For example, consider the situation in the first row of the table where the decisional skills are $(0.8,0.7,0.6,0.5,0.5)$ and the optimal rule is the expert rule $f_{a}$ and, in turn, all payment should be given to individual 1 . If in this situation the decisional skill of individual 2 increases from 0.7 to 0.75 , see the second row, he now deserves $35.48 \%$ of the total payment, because the optimal decision rule changes from $f_{a}$ to $f_{b}$. To further illustrate the possible substantial benefit of investment in skills, suppose that initially all group members share the same skill 0.51 . If one skill changes to 0.535 , that is the vector of competencies becomes $(0.535$, $0.51,0.51,0.51,0.51)$, the optimal rule becomes $f_{e}$ defined by the weights $(3,1,1,1,1)$. If the same skill changes from 0.51 to 0.54 , then the optimal rule becomes $f_{a}$ defined by the weights $(1,0,0,0,0)$. In the former case the justified share in the total payment of 
the group member whose skill has increased changes from $20 \%$ to $63.71 \%$. In the latter case, his share increases from $20 \%$ to $100 \%$.

Notice that in this section, we have focused on the optimal decision rule and, therefore, on the optimal decisional weights which determine by the committees skills. In general, the skill-dependent power is valid when the decision rule is not optimal. Of course, the assignment of decisional weights is not necessarily based on qualifications. Clearly, once weights are based on alternative criteria, such as gender, nepotism or randomness, the existence of unequal contributions and, in turn, payments is inefficient. In particular, women's limited access to organizational power structures may result in inefficiency as well as explain male-female unequal contributions and, in turn, wages.

\section{Conclusion}

In the context of collective decision making, the justified distribution of remunerations can be calculated. It hinges on the applied decision rule, which in our setting is optimally determined on the basis of the group members' decisional skills. Variability in decisional competencies may result in interesting and surprising value distributions that sometimes justify extremely unequal remunerations.

\section{References}

Austen-Smith, D. and J. Banks (1996), "Information aggregation, rationality and the Condorcet jury theorem", American Political Science Review, 90(1), 34-45.

Banzhaf, J.F., (1965), "Weighted Voting Doesn't Work: A Mathematical Analysis", Rutgers Law Review, 19 (2), 317-343.

Ben-Yashar, R. and S. Nitzan (1997), "The optimal decision rule for fixed size committees in dichotomous choice situations - The general result", International Economic Review, 38(1), 175-187. 
Dietrich, F. and C. List (2013), "Propositionwise judgment aggregation: the general case", Social Choice and Welfare, 40, 1067-1095.

Nitzan, S. and J. Paroush (1982), "Optimal decision rules in uncertain dichotomous choice situations", International Economic Review, 23, 289-297.

Nitzan, S. and J. Paroush (2018), "Aggregation of Information by Binary Voting Rules", Chapter 7 in The Oxford Handbook of Public Choice, Volume 1, edited by R.D. Congleton,B.N. Grofman and S.Voigt, Oxford University Press.

Shapley, L. and B. Grofman (1984), "Optimizing group judgmental accuracy in the presence of interdependence", Public Choice, 43, 329-343.

Young, P. (1988), "Condorcet's Theory of Voting", American Political Science Review, 82(4), 1231-1244. 